\title{
Are Obesity Indices Useful for Detecting Subclinical Atheromatosis in a Middle-Aged Population?
}

\author{
Enric Sánchez ${ }^{a} \quad$ Marta Sánchez $^{a}$ Àngels Betriu ${ }^{b}$ Ferran Rius $^{a}$ \\ Gerard Torres $^{c, d}$ Francesc Purroye Reinald Pamplona ${ }^{f}$ \\ Marta Ortega ${ }^{g}$ Carolina López-Cano ${ }^{a}$ Marta Hernández ${ }^{a}$ \\ Marta Bueno $^{a}$ Elvira Fernández ${ }^{\mathrm{b}}$ Javier Salvador ${ }^{\text {h, }}$ i \\ Albert Lecube ${ }^{a, j} \quad$ ILERVAS project collaborators \\ aEndocrinology and Nutrition Department, University Hospital Arnau de Vilanova, Obesity, \\ Diabetes and Metabolism (ODIM) Research Group, IRBLleida, University of Lleida, Lleida, \\ Spain; ${ }^{b}$ Unit for the Detection and Treatment of Atherothrombotic Diseases (UDETMA \\ V\&R), University Hospital Arnau de Vilanova, Vascular and Renal Translational Research \\ Group, IRBLleida, University of Lleida, Lleida, Spain; ' Respiratory Department, University \\ Hospital Arnau de Vilanova-Santa María, Translational Research in Respiratory Medicine, \\ IRBLleida, University of Lleida, Lleida, Spain; ${ }^{\mathrm{d} C e n t r o}$ de Investigación Biomédica en Red \\ de Enfermedades Respiratorias (CIBERES), Instituto de Salud Carlos III (ISCIII), Madrid, \\ Spain; eStroke Unit, University Hospital Arnau de Vilanova. Clinical Neurosciences Group, \\ IRBLleida, University of Lleida, Lleida, Spain; ${ }^{\mathrm{f}}$ Experimental Medicine Department, IRBLleida, \\ University of Lleida, Lleida, Spain; 9 Institut Català de la Salut, Unitat de Suport a la Recerca \\ Lleida, Institut Universitari d'Investigació en Atenció Primària Jordi Gol (IDIAP Jordi

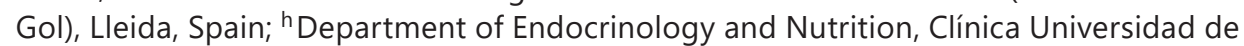 \\ Navarra, Pamplona, Spain; 'Centro de Investigación Biomédica en Red de Fisiopatología \\ de la Obesidad y Nutrición (CIBEROBN), Instituto de Salud Carlos III (ISCIII), Madrid, Spain; \\ jCentro de Investigación Biomédica en Red de Diabetes y Enfermedades Metabólicas \\ Asociadas (CIBERDEM), Instituto de Salud Carlos III (ISCIII), Madrid, Spain
}

\section{Keywords}

Obesity indices $\cdot$ Adipose tissue $\cdot$ Excess body weight $\cdot$ Subclinical atheromatosis

\begin{abstract}
Objective: There is a close relationship between excess adiposity and cardiovascular disease. Although body mass index (BMI) is the most used approach to estimate excess weight, other anthropometric indices have been developed to measure total body and abdominal adipos-
\end{abstract}

Enric Sánchez and Marta Sánchez contributed equally to this work.

For a list of ILERVAS project collaborators, see Appendix. 
Sánchez et al.: Can Obesity Indices Detect Subclinical Atheromatosis in the Middle-Aged?

ity. Here, our objective was to assess the usefulness of these anthropometric indices to detect subclinical atheromatous disease. Methods: A cross-sectional study with 6,809 middle-aged subjects (mean age, 57 [53-63] years) with low to moderate cardiovascular risk from the ILERVAS project. Measures of total body fat (BMI, Clínica Universidad de Navarra - Body Adiposity Estimator [CUN-BAE], and Deurenberg's formula) and central adiposity (waist and neck circumferences, conicity index, waist-to-height ratio, Bonora's equation, the A body adiposity index, and body roundness index) were performed in all participants. Bilateral carotid and femoral ultrasound vascular studies allowed the identification of subjects with plaque. Results: All measured indices were significantly higher in males with subclinical carotid or femoral plaques ( $p \leq 0.021$ for all). Also, a positive and significant correlation between all indices and the number of affected territories was found ( $p \leq 0.013$ for all). From the ROC analysis, all measurements identified patients with asymptomatic atheromatosis but none of them helped make clinical decisions. Regarding females, the results were less conclusive. Conclusion: Obesity indices are related to subclinical atheromatosis, especially in men, in a large cohort of middle-aged subjects. However, the indices could not detect the presence of arterial plaque, so, when used in isolation, are unlikely to be decisive.

(C) 2020 The Author(s)

Published by S. Karger AG, Basel

\section{Introduction}

Cardiovascular diseases are responsible for an estimated 124,000 annual deaths in Spain and up to 17.5 million deaths worldwide [1,2], with well-documented evidence for the association between body mass index (BMI) and cardiovascular mortality [3]. In fact, almost half the risk of coronary heart disease and three-quarters of the risk of stroke are mediated by excess body weight [4]. In this way, the carotid adventitial vasa vasorum, in which expansion to the avascular intima precedes the carotid intima-media thickness, was increased in subjects with morbid obesity in comparison with non-obese subjects and decreased after weight loss induced by bariatric surgery [5]. These data reinforce the role of excess weight in the development of atheromatous disease and have become a huge health problem linked to the worldwide increase in obesity prevalence $[6,7]$. However, the current tools available for assessing cardiovascular risk in clinical practice still do not incorporate obesity measures into their equations.

Because of its simplicity and consistency, BMI is widely used in clinical and epidemiological studies to assess the presence of obesity. Nevertheless, the definition of obesity using BMI classification is far from perfect as it fails to provide an accurate measure of the amount and distribution of body fat $[8,9]$. Also, the relationship between BMI and adiposity is clearly influenced by age, gender, and ethnicity [10]. Nowadays, a direct assessment of body adiposity can be achieved using dual-energy X-ray absorptiometry or magnetic resonance imaging (MRI). The former is widely recognized as the gold standard measurement of total adipose tissue whereas MRI and computed tomography (CT) scan better describe abdominal obesity [11]. However, the cost, complexity, and time demands of these well-established approaches limit their widespread use in clinical practice. For this reason, novel anthropometric indices that combine traditional measures have been developed to measure total body and abdominal adiposity [12-18]. However, little is known about the association of these mathematical equations and the presence of subclinical atheromatous disease.

On this basis, this study sought to determine and compare the discriminatory performance of obesity indices to better estimate the risk of subclinical atheromatosis. For this purpose, we applied three indices of total body fat plus seven indices of abdominal obesity in a large Spanish population with low to moderate cardiovascular risk. Also, all subjects underwent a bilateral carotid and femoral ultrasound exploration to assess the presence of atheromatous disease. 
Sánchez et al.: Can Obesity Indices Detect Subclinical Atheromatosis in the Middle-Aged?

\section{Methods}

\section{Design of the Study and Description of the Study Population}

A total of 6,809 subjects were enrolled between January 2015 and December 2017 from thirty-one primary health care centres in the ILERVAS project, an ongoing prospective study dealing with occult atheromatous disease in the province of Lleida, Spain (ClinTrials.gov Identifier: NCT03228459) [19]. The inclusion criteria were as follows: age 45-70 years, no history of cardiovascular disease, and at least one cardiovascular risk factor (hypertension, smoking, obesity, dyslipidaemia, or a first-degree relative with premature cardiovascular disease). The exclusion criteria were: any type of diabetes, chronic kidney disease, active neoplasia, a life expectancy of less than 18 months, and pregnancy.

\section{Assessment of Obesity-Related Parameters}

Participants were placed in light clothing and without shoes. Weight and height were measured using standard medical equipment to the nearest $0.5 \mathrm{~kg}$ and $1.0 \mathrm{~cm}$, respectively. BMI was calculated as the ratio of body weight $(\mathrm{kg})$ to the height $(\mathrm{m})$ squared. A nonstretchable tape with an accuracy of $0.1 \mathrm{~cm}$ was used to evaluate waist and neck circumferences. Waist circumference was measured midway between the lowest rib and the iliac crest on the horizontal plane with the subject in a standing position. Neck circumference was measured in a plane as horizontal as possible, at a point just below the larynx.

Two equations were added to BMI to estimate total body fat: the Clínica Universidad de Navarra - Body Adiposity Estimator (CUN-BAE) and the formula proposed by Deurenberg et al. $[12,13]$. The CUN-BAE was calculated as follows: $-44.988+(0.503 \times$ age $)+(10.689 \times$ sex $)+$ $(3.172 \times \mathrm{BMI})-\left(0.026 \times \mathrm{BMI}^{2}\right)+(0.181 \times \mathrm{BMI} \times \mathrm{sex})-(0.02 \times \mathrm{BMI} \times \mathrm{age})-\left(0.005 \times \mathrm{BMI}^{2} \times\right.$ sex $)+\left(0.00021 \times \mathrm{BMI}^{2} \times\right.$ age $)$, where male $=0$ and female $=1$ for sex, and age $=$ number of years. The Deurenberg equation assesses body fat using the formula: $(1.20 \times \mathrm{BMI})+(0.23 \times$ age) $-(10.8 \times$ sex $)-5.4$, where sex is 0 for females and 1 for males.

Five equations were added to the waist and neck circumferences to estimate central adiposity: the conicity index proposed by Valdez [14] (1991), the waist-to-height ratio [15], the Bonora equation [16], the A body adiposity index developed by Krakauer and Krakauer [17] (2012), and the recently developed body roundness index proposed by Thomas et al. [18]. The conicity index is based on the hypothesis that people that accumulate abdominal fat have a silhouette like a double cone (that is, two cones sharing the same base, one positioned over the other), whereas subjects with less visceral fat have the shape of a cylinder [14]. Therefore, conicity index ranges from 1.73 (a perfect double cone) to 1.0 (a perfect cylinder) and was obtained following the equation: $0.109^{-1} \times$ waist circumference $(\mathrm{m}) \times(\text { weight }[\mathrm{kg}] / \text { height }[\mathrm{m}])^{-1 / 2}[14]$.

The waist-to-height ratio was calculated as waist circumference $(\mathrm{m})$ divided by height $(\mathrm{m})$ [15]. The equation proposed by Bonora et al. [16] uses different formulas according to gender: $-453.7+(6.37 \times$ waist circumference $)$ for men, and $-370.5+(4.04 \times$ waist circumference $)+$ $(2.62 \times$ age $)$ for women. To obtain the body shape index the eccentricity of the body $(\varepsilon)$ is first determined [17]. The $\varepsilon$ is a non-dimensional value that quantifies the degree of circularity of an ellipse, ranges from 0 (perfect circle) to 1 (a vertical line), and is calculated by the formula: $\left(1-\pi^{-2} \times \text { waist circumference }[\mathrm{m}]^{2} \times \text { height }[\mathrm{m}]^{-2}\right)^{1 / 2}$. Subsequently, the next formula calculates the index: $364.2-(365.5 \times \varepsilon)$, in which values closer to 1 are related to rounder individuals, whereas lower values are associated with leaner individuals [17]. Finally, the body roundness index was calculated as: waist circumference $(\mathrm{m}) /\left(\mathrm{BMI}^{2 / 3} \times\right.$ height $\left.(\mathrm{m})^{1 / 2}\right)[18]$.

\section{Assessment of Atheromatous Disease}

Bilateral carotid (common artery, bifurcation, internal and external) and femoral (common and superficial) areas were explored by a single group of trained sonographers [20]. The ultra- 
Sánchez et al.: Can Obesity Indices Detect Subclinical Atheromatosis in the Middle-Aged?

Table 1. Main clinical and metabolic data of the study population according to the presence of subclinical atheromatosis

\begin{tabular}{lccc}
\hline & No atheromatosis & Subclinical atheromatosis & $p$ value \\
\hline Total $n$ & 2,132 & 4,677 & - \\
Women & $1,388(65.1)$ & $2,099(44.9)$ & $<0.001$ \\
Age, years & $56[52-61]$ & $58[53-63]$ & $<0.001$ \\
Current or former smoker & $998(46.8)$ & $3,000(64.1)$ & $<0.001$ \\
Blood hypertension & $712(33.4)$ & $2,019(43.2)$ & $<0.001$ \\
Systolic BP, mm Hg & $126[116-138]$ & $132[121-143]$ & $<0.001$ \\
Diastolic BP, mm Hg & $80[74-86]$ & $82[76-88]$ & $<0.001$ \\
Pulse pressure, mm Hg & $46[39-54]$ & $49[42-58]$ & $<0.001$ \\
Dyslipidaemia & $1,012(47.5)$ & $2,557(54.7)$ & $<0.001$ \\
Total cholesterol, mg/dL & $202[179-228]$ & $204[181-230]$ & 0.044 \\
Obesity & $671(31.5)$ & $1,371(29.3)$ & 0.071 \\
BMI, kg/m & $28.6[25.9-32.0]$ & $28.5[25.3-32.0]$ & 0.088 \\
\hline
\end{tabular}

Data are expressed as $n(\%)$ or median [interquartile range]. BP, blood pressure; BMI, body mass index.

sound study was done using an ultrasonic Doppler Ultrasound Vivid-I (GE Healthcare, Waukesha, WI, USA) equipped with a linear transducer broadband linear 12L-RS/4-13 MHz. Standardized and validated scanning and reading protocols were used [20]. A plaque was defined as focal intima-media intrusion into the lumen $\geq 1.5 \mathrm{~mm}$ thick, and subclinical atheromatosis was defined as the presence of at least one plaque in any of the twelve assessed areas $[21,22]$. The number of affected territories, ranging from 0 to 12 , was noted.

\section{Covariate Assessment}

The smoking status (never/current/former) was recorded. Smokers who stopped smoking $\geq 1$ year prior to recruitment were considered former smokers. Blood pressure was measured in triplicate, after a 5-min rest using an automated device (Omron M6 Comfort HEM-7221-E; Omron Healthcare, Kyoto, Japan) at 2-min intervals, and the mean of the last two was calculated. Pulse pressure, subtracting the diastolic from the systolic blood pressure, was also assessed. Finally, a dried capillary blood sample for total cholesterol (mg/dL) measurement was performed using a REFLOTRON ${ }^{\circledR}$ (Roche Diagnostics, GmbH, Germany).

\section{Statistical Analysis}

Subjects were classified based on the diagnosis of subclinical atheromatosis. The normal distribution of the variables was evaluated using the Shapiro-Wilk test. Given its skewed distribution, quantitative data are expressed as the median (interquartile range). Baseline characteristics across the presence of subclinical atheromatous disease were compared by the MannWhitney U test for quantitative variables, and the Pearson $\chi^{2}$ test for categorical variables. The relationships between obesity indices were assessed by the Spearman correlation test.

The areas under receiver operating characteristic (ROC) curves with a complete sensitivity/ specificity report were calculated to examine the diagnostic ability of each adiposity measure to discriminate subclinical atheromatosis. An odds ratio with its $95 \%$ confidence interval was finally calculated. The total area under the ROC curve was interpreted using the following guidelines: $0.9-1.0$, excellent; $0.8-0.9$, good; $0.7-0.8$, fair; $0.6-0.7$, poor; and $0.5-0.6$, not useful.

All statistical analyses were performed using SSPS statistical package (SPSS Statistics for Windows, Version 20.0; IBM, Armonk, NY, USA). All $p$ values were based on a two-sided test of statistical significance, set at $p<0.05$. 
Sánchez et al.: Can Obesity Indices Detect Subclinical Atheromatosis in the Middle-Aged?

Table 2. Results of the obesity indices in male and female participants according to the presence of subclinical atheromatosis

\begin{tabular}{|c|c|c|c|}
\hline & No atheromatosis & Subclinical atheromatosis & $p$ value \\
\hline \multicolumn{4}{|l|}{ Male subjects } \\
\hline$n$ & 744 & 2,578 & - \\
\hline \multicolumn{4}{|l|}{ Total body fat } \\
\hline BMI, $\mathrm{kg} / \mathrm{m}^{2}$ & $28.4[25.8-31.4]$ & $28.8[26.4-31.8]$ & 0.021 \\
\hline CUN-BAE, \% & $29.8[26.1-33.4]$ & $30.6[27.4-34.1]$ & $<0.001$ \\
\hline Deurenberg, \% & $30.2[26.8-33.9]$ & $31.4[28.1-35.2]$ & $<0.001$ \\
\hline \multicolumn{4}{|l|}{ Central adiposity } \\
\hline Waist circumference, $\mathrm{cm}$ & 101 [94-108] & $102[96-110]$ & 0.003 \\
\hline Conicity index & $1.32[1.28-1.36]$ & $1.33[1.30-1.38]$ & $<0.001$ \\
\hline Waist-to-height ratio & $0.58[0.55-0.63]$ & $0.60[0.56-0.64]$ & $<0.001$ \\
\hline Bonora, $\mathrm{cm}^{2}$ & 189.7 [145.1-234.3] & $196.0[157.8-247.0]$ & 0.003 \\
\hline A body shape index & $0.083[0.080-0.084]$ & $0.083[0.081-0.085]$ & $<0.001$ \\
\hline Body roundness index & $5.01[4.23-6.06]$ & $5.34[4.51-6.31]$ & $<0.001$ \\
\hline Neck circumference, $\mathrm{cm}$ & $40.5[39.0-42.5]$ & $41.0[39.0-43.0]$ & 0.004 \\
\hline \multicolumn{4}{|l|}{ Female subjects } \\
\hline$n$ & 1,388 & 2,099 & - \\
\hline \multicolumn{4}{|l|}{ Total body fat } \\
\hline BMI, $\mathrm{kg} / \mathrm{m}^{2}$ & $28.6[25.1-32.3]$ & $28.4[25.2-32.2]$ & 0.772 \\
\hline CUN-BAE, \% & $42.2[38.2-46.3]$ & $42.3[38.5-46.2]$ & 0.351 \\
\hline Deurenberg, \% & $31.5[27.3-36.5]$ & $32.0[27.9-36.4]$ & 0.039 \\
\hline \multicolumn{4}{|l|}{ Central adiposity } \\
\hline Waist circumference, $\mathrm{cm}$ & 98 [90-106] & 99 [92-107] & 0.120 \\
\hline Conicity index & $1.35[1.30-1.40]$ & $1.36[1.31-1.41]$ & $<0.001$ \\
\hline Waist-to-height ratio & $0.63[0.58-0.68]$ & $0.63[0.58-0.69]$ & 0.077 \\
\hline Bonora, $\mathrm{cm}^{2}$ & 180.2 [147.1-215.9] & 189.1 [155.1-224.9] & 0.076 \\
\hline A body shape index & $0.084[0.081-0.087]$ & $0.085[0.082-0.088]$ & $<0.001$ \\
\hline Body roundness index & $6.00[4.90-7.25]$ & $6.09[4.98-7.40]$ & $<0.001$ \\
\hline Neck circumference, $\mathrm{cm}$ & $35.0[33.0-36.5]$ & $35.0[35.5-37.0]$ & 0.001 \\
\hline
\end{tabular}

Data are expressed as median [interquartile range]. BMI, body mass index; CUN-BAE, Clínica Universidad de Navarra - Body Adiposity Estimator.

\section{Results}

The prevalence of subclinical atheromatous disease in the study population was $68.6 \%$. The main clinical and metabolic data of the study population according to the presence of at least one plaque are shown in Table 1. Atheromatosis was higher in smokers and men, and increased with age, blood pressure levels, and total cholesterol concentration. However, no differences in the prevalence of obesity according to BMI (29.3 vs. $31.5 \%, p=0.071$ ) or in BMI values $\left(28.5[25.3-32.0]\right.$ vs. $\left.28.6[25.9-32.0] \mathrm{kg} / \mathrm{m}^{2}, p=0.088\right]$ were observed when comparing participants with and without subclinical atheromatous disease.

Table 2 displays the results of the obesity indices related to total body fat and central adiposity according to gender in participants with and without atheromatous disease. All measured indices were significantly higher in male individuals with subclinical atheromatosis in comparison with subjects without carotid or femoral plaques ( $p \leq 0.021$ for all). However, when female participants were evaluated, we failed to find significant differences in indices related with total body fat, such as BMI and CUN-BAE, and abdominal adiposities, such as waist circumference, waist-to-height ratio, and Bonora's equation.

In male subjects, a positive and significant correlation between all indices for fat accumulation and the number of arterial territories with plaque was found in the bivariate analysis 
Sánchez et al.: Can Obesity Indices Detect Subclinical Atheromatosis in the Middle-Aged?

Table 3. Correlations of obesity indices with the number of affected territories with plaque according to gender distribution

\begin{tabular}{|c|c|c|c|c|}
\hline & \multicolumn{2}{|c|}{ Male subjects } & \multicolumn{2}{|c|}{ Female subjects } \\
\hline & $r$ & $p$ & $r$ & $p$ \\
\hline BMI $\left(\mathrm{kg} / \mathrm{m}^{2}\right)$ & 0.043 & 0.013 & -0.011 & 0.528 \\
\hline CUN-BAE (\%) & 0.077 & $<0.001$ & 0.012 & 0.473 \\
\hline Deurenberg (\%) & 0.117 & $<0.001$ & 0.033 & 0.054 \\
\hline Waist circumference $(\mathrm{cm})$ & 0.064 & $<0.001$ & 0.028 & 0.094 \\
\hline Conicity index & 0.130 & $<0.001$ & 0.092 & $<0.001$ \\
\hline Waist-to-height ratio & 0.105 & $<0.001$ & 0.030 & 0.081 \\
\hline Bonora $\left(\mathrm{cm}^{2}\right)$ & 0.063 & $<0.001$ & 0.087 & $<0.001$ \\
\hline A body shape index & 0.131 & $<0.001$ & 0.100 & $<0.001$ \\
\hline Body roundness index & 0.105 & $<0.001$ & 0.030 & 0.080 \\
\hline Neck circumference $(\mathrm{cm})$ & 0.066 & $<0.001$ & 0.063 & $<0.001$ \\
\hline
\end{tabular}

BMI, body mass index; CUN-BAE, Clínica Universidad de Navarra - Body Adiposity Estimator.

( $p \leq 0.013$ for all comparisons) (Table 3). Nevertheless, in female participants, the correlation with total affected territories was only significant for the conicity index, Bonora's equation, the A body shape index, and neck circumference ( $p<0.001$ for all comparisons).

From the ROC analysis, we show that in male participants, all measurements related to either total body or abdominal adiposity were useful indicators to identify patients with asymptomatic atheromatosis (Table 4). However, the total area under the ROC curve indicates that none of them is helpful for taking clinical decisions in our population. When the ROC analysis was applied to female participants, although Deurenberg's index, conicity index, the A body shape index, and neck circumference were able to discriminate individuals with atheromatous disease, none of them appeared to be of clinical interest in the identification of vulnerable subjects.

\section{Discussion}

This study compares the relationship between different equations used to estimate body fat and abdominal obesity and the presence of subclinical atheromatosis in a middle-aged Caucasian cohort. Our data support the hypothesis that excess body fat is related to the presence of at least one plaque in any of the twelve assessed areas in carotid and femoral arteries. However, we should also note three main findings from our work: (i) a marked difference between the two genders, suggesting that sex-specific values should be used in clinical practice when attempting to evaluate adiposity accumulation in a general population; (ii) the absence of clear differences between indices related to total body or abdominal adiposity to better discriminate the presence of subclinical atheromatosis; and (iii) no isolated obesity index appears to be good enough to be incorporated in clinical practice to identify subjects at risk.

The relationship between obesity and cardiovascular diseases is well known and is predominantly related to the visceral accumulation of fat depots. Visceral fat area quantified by MRI or dual-source CT has been shown to effectively identify carotid atherosclerosis, especially in Asian populations [23-25]. When the visceral fat area was measured in 3,381 participants older than 40 years without a previous history of cardiovascular diseases, a clear and independent association with carotid intima-media thickness was observed [23]. Also, when waist circumference was used as a surrogate of abdominal adiposity, similar results were 
Sánchez et al.: Can Obesity Indices Detect Subclinical Atheromatosis in the Middle-Aged?

Table 4. ROC curves and appropriate cut-off of adiposity indexes in subclinical atheromatous disease prediction among male and female subjects

\begin{tabular}{|c|c|c|c|c|c|c|}
\hline & Cut-off & Sensitivity & Specificity & AUROC & $95 \% \mathrm{CI}$ & $p$ \\
\hline \multicolumn{7}{|l|}{ Male subjects } \\
\hline BMI, $\mathrm{kg} / \mathrm{m}^{2}$ & 26.5 & 0.740 & 0.690 & 0.528 & $0.504-0.552$ & 0.021 \\
\hline CUN-BAE, \% & 26.3 & 0.814 & 0.743 & 0.545 & $0.521-0.569$ & $<0.001$ \\
\hline Deurenberg, \% & 26.3 & 0.852 & 0.770 & 0.565 & $0.541-0.589$ & $<0.001$ \\
\hline Waist circumference, $\mathrm{cm}$ & 94.5 & 0.777 & 0.712 & 0.536 & $0.513-0.560$ & 0.002 \\
\hline Conicity index & 1.36 & 0.383 & 0.272 & 0.566 & $0.543-0.589$ & $<0.001$ \\
\hline Waist-to-height ratio & 0.59 & 0.561 & 0.456 & 0.560 & $0.536-0.584$ & $<0.001$ \\
\hline Bonora, $\mathrm{cm}^{2}$ & 148.3 & 0.777 & 0.712 & 0.536 & $0.513-0.560$ & 0.002 \\
\hline Body roundness index & 5.15 & 0.558 & 0.452 & 0.560 & $0.536-0.584$ & $<0.001$ \\
\hline A body shape index & 0.085 & 0.316 & 0.207 & 0.561 & $0.538-0.584$ & $<0.001$ \\
\hline Neck circumference, cm & 41.3 & 0.442 & 0.380 & 0.534 & $0.511-0.588$ & 0.012 \\
\hline \multicolumn{7}{|l|}{ Female subjects } \\
\hline BMI, $\mathrm{kg} / \mathrm{m}^{2}$ & 26.5 & 0.656 & 0.638 & 0.497 & $0.477-0.517$ & 0.772 \\
\hline CUN-BAE, \% & 35.1 & 0.908 & 0.886 & 0.509 & $0.490-0.529$ & 0.351 \\
\hline Deurenberg, \% & 30.1 & 0.628 & 0.574 & 0.521 & $0.501-0.541$ & 0.036 \\
\hline Waist circumference, $\mathrm{cm}$ & 90.5 & 0.775 & 0.738 & 0.516 & $0.496-0.535$ & 0.121 \\
\hline Conicity index & 1.36 & 0.539 & 0.452 & 0.548 & $0.529-0.568$ & $<0.001$ \\
\hline Waist-to-height ratio & 0.65 & 0.417 & 0.385 & 0.518 & $0.498-0.537$ & 0.073 \\
\hline Bonora, $\mathrm{cm}^{2}$ & 176.3 & 0.606 & 0.528 & 0.548 & $0.528-0.567$ & $<0.001$ \\
\hline Body roundness index & 4.30 & 0.884 & 0.850 & 0.518 & $0.498-0.538$ & 0.072 \\
\hline A body shape index & 0.085 & 0.520 & 0.446 & 0.552 & $0.533-0.572$ & $<0.001$ \\
\hline Neck circumference, cm & 34.8 & 0.561 & 0.503 & 0.534 & $0.514-0.554$ & 0.001 \\
\hline
\end{tabular}

AUROC, area under the receiver operating characteristic curve; BMI, body mass index; CUN-BAE, Clínica Universidad de Navarra - Body Adiposity Estimator.

communicated not only in Asian participants but also in Europeans [26, 27]. In this way, waist circumference, together with serum concentrations of low-density lipoprotein cholesterol and insulin, was directly associated with the 6-year progression of carotid intima-media thickness in a population of 1,809 young adults from Finland [26]. Similarly, waist circumference was significantly related to carotid intima-media thickness in 518 postmenopausal Chinese women aged 50-64 years, independent of age, hormone therapy, sociodemographic factors, and BMI [27]. Our results, assessing several indices of total body fat and abdominal obesity in a large Spanish population with low to moderate cardiovascular risk, support the association of excess adiposity stores with subclinical atherosclerosis. However, the magnitude of this association is weak and precludes the application of obesity indices alone to screen subjects at risk of suffering cardiovascular events.

Our study also reinforces the concept that obesity indices are gender specific. The reasons for gender disparity are unclear, although differences in regional adipose tissue distribution and body fatness are well documented [28]. In fact, all tested indices in males included in the ILERVAS project appear to be significantly higher in those with atheromatous disease compared to subjects without plaque. However, only one of the three indices that estimate total body fat and four of the seven indices that assess abdominal adiposity follow this pattern among women. Data from 1,005 middle-aged Chinese individuals (49\% women) free of cardiovascular disease who underwent MRI showed that visceral fat adiposity effectively identified carotid atherosclerosis in men but not in women [25]. However, among 3,001 participants from the Framingham Heart Study (52\% women) free of clinical cardiovascular disease, relationships between the visceral adipose tissue assessed by multidetector CT and metabolic risk factors were consistently stronger in women than in men [29]. Also, in the 
Sánchez et al.: Can Obesity Indices Detect Subclinical Atheromatosis in the Middle-Aged?

Jackson Heart Study, with 2,477 participants (64\% women), the association of visceral and subcutaneous adipose tissue with most of the evaluated risk factors was stronger for women than for men [30].

Previous studies have evaluated the relation of obesity indices with the presence of vascular risk factors rather than their capacity to discriminate subjects with atheromatous disease. In this way, our study demonstrates that the estimation of total body fat with the Deurenberg equation, as well as the calculation of central adiposity with the conicity index, the body roundness index, and the A body shape index can identify subjects with at least one carotid or femoral plaque in both genders. The prediction of the percentage of body fat using the age- and sex-specific equation proposed in 1991 by Deurenberg et al. [13] takes for granted that, when the BMI increases over a certain threshold, the excess value is for a fixed part due to body fat. This equation correlated well with body fat measured by either dualenergy X-ray absorptiometry or densitometry (underwater weighing) in a cross-sectional observational study that included 234 females and 182 males [31]. However, no previous data about its relationship with vascular diseases have been communicated.

The conicity index does not include gender-discriminating measures for the estimation of central obesity. Although its measurement was associated with type 2 diabetes and hypertension in Brazilian women, it failed to correlate with the plasma atherogenic index in 414 sedentary young males from Nigeria or in 909 relatively lean patients in maintenance haemodialysis from China [32-34]. In addition, the conicity index did not show a positive association with the presence of stenosis in major coronary arteries in 610 subjects from Iran [35]. The low ability of this index to predict vascular disease has been justified because weight might dilute the influence of height according to its formula [36]. Therefore, our results present the first positive data supporting the role of the conicity index in low to moderate cardiovascular risk populations, albeit its total area under the ROC curve suggests it as not useful.

The predictive capability of the body roundness index for identifying cardiovascular diseases was first assessed by Maessen et al. [37] in 4,627 Dutch participants of the Nijmegen Exercise Study. In this cross-sectional study, when comparing the lowest with the highest quintile of the index, the adjusted OR $(95 \% \mathrm{CI})$ to identify the previous history of myocardial infarction and stroke showed a 3-fold significant increase but was not superior to BMI or waist circumference. On the other hand, the A body shape index was more predictive for premature mortality risk across age, sex, and weight than either waist circumference or BMI in the USA population from the National Health and Nutrition Examination Survey (NHANES) 1999-2004 [17]. In another national survey, the British Health and Lifestyle Survey (HALS), the A body shape index was a consistent predictor of mortality hazard over at least 20 years of follow-up, and participants who experienced a rise in their index were at greater risk than those who experienced a decrease [38]. Similarly, in a 13-year follow-up cohort study with 41,020 Spanish adults, the A body shape index, waist circumference, and waist-to-hip ratio, but not BMI, were significantly associated with the total incidence of stroke in men [39].

Finally, neck circumference is considered a promising marker not only for upper-body subcutaneous fat but also for visceral obesity $[40,41]$. In fact, its measurement was positively associated with visceral fat among the 1,053 adults included in the Brazilian Metabolic Syndrome Study and has shown a strong correlation with 10-year coronary artery disease risk as calculated by the Framingham risk score [42, 43]. However, neck circumference was not associated with advanced atherosclerosis in 120 subjects who underwent coronary angiography and carotid Doppler ultrasound [44]. Our data also failed to identify neck circumference as a better predictor of subclinical atherosclerosis than the rest of the obesity indices.

Some potential limitations must be underlined in our study. First, and probably the main one, is that we lack the true percentage and distribution of body fat determined with a gold standard tool. There is accumulating evidence that once visceral adipose tissue increases, 
Sánchez et al.: Can Obesity Indices Detect Subclinical Atheromatosis in the Middle-Aged?

dyslipidaemia and endothelial dysfunction remain as additive risk factors in the promotion of atherosclerosis [29, 30,45]. However, the close relationship between obesity indices and adiposity has been previously well established, and remarkable differences between them have not been detected in our large population. These findings are not especially novel. However, the use of so many of the proposed indices allows a clear evaluation of the comparative clinical value of such indices. Second, we have not evaluated other obesity indices including hip circumference, abdominal volume index, body adiposity index, and waist-to-hip ratio. We are not sure whether their inclusion would improve our results. Third, as an observational and cross-sectional study, a causal relationship between excess body adiposity and subclinical atheromatosis cannot be established.

In conclusion, our data show that obesity indices are related to subclinical atheromatosis, especially in men, in a large cohort of middle-aged subjects with low to moderate cardiovascular risk. However, the indices could not detect the presence of arterial plaque, so its applicability in clinical practice in an isolated way might be not decisive. Global cardiovascular risk assessment to identify the most vulnerable subjects at risk of suffering an event is far from perfect, and all involved factors need to be considered globally.

\section{Appendix}

\section{ILERVAS Project Collaborators}

José Manuel Valdivielso (University of Lleida, Lleida, Spain), Marcelino Bermúdez-López (University of Lleida, Lleida, Spain), Montse Martínez-Alonso (University of Lleida, Lleida, Spain), Manuel Sánchez-de-la-Torre (University of Lleida, Lleida, Spain; Instituto de Salud Carlos III (ISCIII), Madrid, Spain), Dinora Polanco (University of Lleida, Lleida, Spain), Ferrán Barbé (University of Lleida, Lleida, Spain; Instituto de Salud Carlos III (ISCIII), Madrid, Spain), Guillermo Suárez (University of Lleida, Lleida, Spain; Instituto de Salud Carlos III (ISCIII), Madrid, Spain), Laura Colàs-Campàs (University of Lleida, Lleida, Spain), Ikram Benabdelhak Manuel Portero-Otin (University of Lleida, Lleida, Spain), Mariona Jové (University of Lleida, Lleida, Spain), Eva Miquel (Balaguer Primary Health Care Unit, Lleida, Spain), Cristina Farràs (Borges Blanques Primary Health Care Unit, Lleida, Spain).

\section{Acknowledgments}

This work was supported by grants from the Diputació de Lleida and Generalitat de Catalunya (2017SGR696 and SLT0021600250) and Menarini Spain S.A. CIBER de Diabetes y Enfermedades Metabólicas Asociadas and CIBER de Enfermedades Respiratorias are initiatives of the Instituto de Salud Carlos III. The authors would also like to thank Fundació Renal Jaume Arnó, Eva Castro, Virtudes María, Marta Elias, Teresa Vidal, Ma del Valle Peña, Cristina Dominguez, Noemi Nova, Alba Prunera, Núria Sans, Meritxell Soria, and the Primary Care teams from Lleida for recruiting subjects and for their efforts in the accurate development of the ILERVAS project.

\section{Statement of Ethics}

Informed and written consent was obtained from all participants, and the protocol was approved by the Arnau de Vilanova University Hospital ethics committee (CEIC-1410). The study was conducted according to the ethical guidelines of the Helsinki Declaration and Spanish legislation regarding the protection of personal data was also followed. 
Sánchez et al.: Can Obesity Indices Detect Subclinical Atheromatosis in the Middle-Aged?

\title{
Disclosure Statement
}

\author{
The authors declare no conflict of interest.
}

\section{References}

1 Instituto Nacional de Estadística - INE. Defunciones según la causa de muerte. Available from: http://www. ine.es/prensa/edcm_2015.pdf.

2 Global Health Estimates. Deaths, disability-adjusted life year (DALYs), years of life lost (YLL) and years lost due to disability (YLD) by cause, age and sex, 2000-2012. Geneva: World Health Organization. Available from: http://www.who.int/healthinfo/global_burden_disease/estimates/en/.

3 Twig G, Yaniv G, Levine H, Leiba A, Goldberger N, Derazne E, et al. Body-mass index in 2.3 million adolescents and cardiovascular death in adulthood. N Engl J Med. 2016 Jun;374(25):2430-40.

4 Lu Y, Hajifathalian K, Ezzati M, Woodward M, Rimm EB, Danaei G; Global Burden of Metabolic Risk Factors for Chronic Diseases Collaboration (BMI Mediated Effects). Metabolic mediators of the effects of body-mass index, overweight, and obesity on coronary heart disease and stroke: a pooled analysis of 97 prospective cohorts with 1.8 million participants. Lancet. 2014 Mar;383(9921):970-83.

5 Rius F, Sánchez E, Betriu À, Baena-Fustegueras JA, Yeramian A, Vidal T, et al. Influence of morbid obesity and bariatric surgery impact on the carotid adventitial vasa vasorum signal. Obes Surg. 2018 Dec;28(12):3935-42.

6 NCD Risk Factor Collaboration (NCD-RisC). Trends in adult body-mass index in 200 countries from 1975 to 2014: a pooled analysis of 1698 population-based measurement studies with $19 \cdot 2$ million participants. Lancet. 2016 Apr;387(10026):1377-96.

7 Abarca-Gómez L, Abdeen ZA, Hamid ZA, Abu-Rmeileh NM, Acosta-Cazares B, Acuin C, et al.; NCD Risk Factor Collaboration (NCD-RisC). Worldwide trends in body-mass index, underweight, overweight, and obesity from 1975 to 2016: a pooled analysis of 2416 population-based measurement studies in 128.9 million children, adolescents, and adults. Lancet. 2017 Dec;390(10113):2627-42.

8 Blundell JE, Dulloo AG, Salvador J, Frühbeck G; EASO SAB Working Group on BMI. Beyond BMI - phenotyping the obesities. Obes Facts. 2014;7(5):322-8.

9 Gómez-Ambrosi J, Silva C, Galofré JC, Escalada J, Santos S, Millán D, et al. Body mass index classification misses subjects with increased cardiometabolic risk factors related to elevated adiposity. Int J Obes. 2012 Feb;36(2): 286-94.

10 Okorodudu DO, Jumean MF, Montori VM, Romero-Corral A, Somers VK, Erwin PJ, et al. Diagnostic performance of body mass index to identify obesity as defined by body adiposity: a systematic review and meta-analysis. Int J Obes. 2010 May;34(5):791-9.

11 Kelly TL, Wilson KE, Heymsfield SB. Dual energy X-Ray absorptiometry body composition reference values from NHANES. PLoS One. 2009 Sep;4(9):e7038.

12 Gómez-Ambrosi J, Silva C, Catalán V, Rodríguez A, Galofré JC, Escalada J, et al. Clinical usefulness of a new equation for estimating body fat. Diabetes Care. 2012 Feb;35(2):383-8.

13 Deurenberg P, Weststrate JA, Seidell JC. Body mass index as a measure of body fatness: age- and sex-specific prediction formulas. Br J Nutr. 1991 Mar;65(2):105-14.

14 Valdez R. A simple model-based index of abdominal adiposity. J Clin Epidemiol. 1991;44(9):955-6.

15 Ashwell M, Gunn P, Gibson S. Waist-to-height ratio is a better screening tool than waist circumference and BMI for adult cardiometabolic risk factors: systematic review and meta-analysis. Obes Rev. 2012 Mar;13(3):27586.

16 Bonora E, Micciolo R, Ghiatas AA, Lancaster JL, Alyassin A, Muggeo M, et al. Is it possible to derive a reliable estimate of human visceral and subcutaneous abdominal adipose tissue from simple anthropometric measurements? Metabolism. 1995 Dec;44(12):1617-25.

17 Krakauer NY, Krakauer JC. A new body shape index predicts mortality hazard independently of body mass index. PLoS One. 2012;7(7):e39504.

18 Thomas DM, Bredlau C, Bosy-Westphal A, Mueller M, Shen W, Gallagher D, et al. Relationships between body roundness with body fat and visceral adipose tissue emerging from a new geometrical model. Obesity (Silver Spring). 2013 Nov;21(11):2264-71.

19 Betriu À, Farràs C, Abajo M, Martinez-Alonso M, Arroyo D, Barbé F, et al. Randomised intervention study to assess the prevalence of subclinical vascular disease and hidden kidney disease and its impact on morbidity and mortality: the ILERVAS project. Nefrologia. 2016 Jul-Aug;36(4):389-96.

20 Sabetai MM, Tegos TJ, Nicolaides AN, Dhanjil S, Pare GJ, Stevens JM. Reproducibility of computer-quantified carotid plaque echogenicity: can we overcome the subjectivity? Stroke. 2000 Sep;31(9):2189-96.

21 Stein JH, Korcarz CE, Hurst RT, Lonn E, Kendall CB, Mohler ER, et al.; American Society of Echocardiography Carotid Intima-Media Thickness Task Force; Endorsed by the Society for Vascular Medicine. Use of carotid ultrasound to identify subclinical vascular disease and evaluate cardiovascular disease risk: a consensus statement from the American Society of Echocardiography Carotid Intima-Media Thickness Task Force. J Am Soc Echocardiogr. 2008 Feb;21(2):93-111. 
Sánchez et al.: Can Obesity Indices Detect Subclinical Atheromatosis in the Middle-Aged?

22 Touboul PJ, Hennerici MG, Meairs S, Adams H, Amarenco P, Desvarieux M, et al.; Advisory Board of the 3rd Watching the Risk Symposium 2004, 13th European Stroke Conference. Mannheim intima-media thickness consensus. Cerebrovasc Dis. 2004;18(4):346-9.

23 Ren C, Zhang J, Xu Y, Xu B, Sun W, Sun J, et al. Association between carotid intima-media thickness and index of central fat distribution in middle-aged and elderly Chinese. Cardiovasc Diabetol. 2014 Oct;13(1):139.

24 Oike M, Yokokawa H, Fukuda H, Haniu T, Oka F, Hisaoka T, et al. Association between abdominal fat distribution and atherosclerotic changes in the carotid artery. Obes Res Clin Pract. 2014 Sep-0ct;8(5):e448-58.

25 Wang Y, Ma X, Zhou M, Zong W, Zhang L, Hao Y, et al. Contribution of visceral fat accumulation to carotid intima-media thickness in a Chinese population. Int J Obes. 2012 Sep;36(9):1203-8.

26 Koskinen J, Kähönen M, Viikari JS, Taittonen L, Laitinen T, Rönnemaa T, et al. Conventional cardiovascular risk factors and metabolic syndrome in predicting carotid intima-media thickness progression in young adults: the cardiovascular risk in young Finns study. Circulation. 2009 Jul;120(3):229-36.

27 Yu RH, Ho SC, Ho SS, Woo JL, Ahuja AT. Association of general and abdominal obesities and metabolic syndrome with subclinical atherosclerosis in asymptomatic Chinese postmenopausal women. Menopause. 2008 Jan-Feb; 15(1):185-92.

28 Després JP, Couillard C, Gagnon J, Bergeron J, Leon AS, Rao DC, et al. Race, visceral adipose tissue, plasma lipids, and lipoprotein lipase activity in men and women: the Health, Risk Factors, Exercise Training, and Genetics (HERITAGE) family study. Arterioscler Thromb Vasc Biol. 2000 Aug;20(8):1932-8.

29 Fox CS, Massaro JM, Hoffmann U, Pou KM, Maurovich-Horvat P, Liu CY, et al. Abdominal visceral and subcutaneous adipose tissue compartments: association with metabolic risk factors in the Framingham Heart Study. Circulation. 2007 Jul;116(1):39-48.

30 Liu J, Fox CS, Hickson DA, May WD, Hairston KG, Carr JJ, et al. Impact of abdominal visceral and subcutaneous adipose tissue on cardiometabolic risk factors: the Jackson Heart Study. J Clin Endocrinol Metab. 2010 Dec; 95(12):5419-26.

31 Deurenberg P, Andreoli A, Borg P, Kukkonen-Harjula K, de Lorenzo A, van Marken Lichtenbelt WD, et al. The validity of predicted body fat percentage from body mass index and from impedance in samples of five European populations. Eur J Clin Nutr. 2001 Nov;55(11):973-9.

32 Andrade MD, Freitas MC, Sakumoto AM, Pappiani C, Andrade SC, Vieira VL, et al. Association of the conicity index with diabetes and hypertension in Brazilian women. Arch Endocrinol Metab. 2016 Oct; 60(5):436-42.

33 Ezeukwu A0, Agwubike E0. Anthropometric measures of adiposity as correlates of atherogenic index of plasma in non-obese sedentary Nigerian males. Libyan J Med. 2014;9(1):23798.

34 Zhou C, Peng H, Yuan J, Lin X, Zha Y, Chen H. Visceral, general, abdominal adiposity and atherogenic index of plasma in relatively lean hemodialysis patients. BMC Nephrol. 2018 Aug;19(1):206.

35 Salari A, Shakiba M, Mahdavi-Roshan M, Gholipour M, Naghshbandi M, Rajabi R. The association between various indices of obesity and severity of atherosclerosis in adults in the north of Iran. Medicine (Baltimore). 2016 Dec;95(50):e5670.

36 Motamed N, Sohrabi M, Poustchi H, Maadi M, Malek M, Keyvani H, et al. The six obesity indices, which one is more compatible with metabolic syndrome? A population-based study. Diabetes Metab Syndr. 2017 Jul - Sep; 11(3):173-7.

37 Maessen MF, Eijsvogels TM, Verheggen RJ, Hopman MT, Verbeek AL, de Vegt F. Entering a new era of body indices: the feasibility of a body shape index and body roundness index to identify cardiovascular health status. PLoS One. 2014 Sep;9(9):e107212.

38 Krakauer NY, Krakauer JC. Dynamic association of mortality hazard with body shape. PLoS One. 2014 Feb; $9(2): e 88793$.

39 Abete I, Arriola L, Etxezarreta N, Mozo I, Moreno-Iribas C, Amiano P, et al. Association between different obesity measures and the risk of stroke in the EPIC Spanish cohort. Eur J Nutr. 2015 Apr;54(3):365-75.

40 Arias Téllez MJ, Martinez-Tellez B, Soto J, Sánchez-Delgado G. [Validity of neck circumference as a marker of adiposity in children and adolescents, and in adults: a systematic review]. Nutr Hosp. 2018 Apr;35(3):707-21. Spanish.

41 Zhao L, Huang G, Xia F, Li Q, Han B, Chen Y, et al. Neck circumference as an independent indicator of visceral obesity in a Chinese population. Lipids Health Dis. 2018 Apr;17(1):85.

42 Stabe C, Vasques AC, Lima MM, Tambascia MA, Pareja JC, Yamanaka A, et al. Neck circumference as a simple tool for identifying the metabolic syndrome and insulin resistance: results from the Brazilian Metabolic Syndrome Study. Clin Endocrinol (Oxf). 2013 Jun;78(6):874-81.

43 Koppad AK, Kaulgud RS, Arun BS. A study of correlation of neck circumference with Framingham Risk Score as a predictor of coronary artery disease. J Clin Diagn Res. 2017 Sep;11(9):0C17-20.

44 Maddaloni E, Cavallari I, De Pascalis M, Keenan H, Park K, Manfrini S, et al. Relation of body circumferences to cardiometabolic disease in overweight-obese Subjects. Am J Cardiol. 2016 Sep;118(6):822-7.

45 Luna-Luna M, Medina-Urrutia A, Vargas-Alarcón G, Coss-Rovirosa F, Vargas-Barrón J, Pérez-Méndez Ó. Adipose tissue in metabolic syndrome: onset and progression of atherosclerosis. Arch Med Res. 2015 Jul; 46(5):392-407. 